\title{
Right Lung Alveolus
}

National Cancer Institute

\section{Source}

National Cancer Institute. Right Lung Alveolus. NCI Thesaurus. Code C49278.

An alveolus located in the right lung. 